\title{
Corela
}

Cognition, représentation, langage

HS-25 | 2018

Les procédés implicites dans l'interface sémantiquepragmatique

\section{L'implicite comme moyen de persuasion : une approche quantitative}

\author{
Edoardo Lombardi Vallauri
}

\section{OpenEdition}

Journals

Édition électronique

URL : http://journals.openedition.org/corela/6112

DOI : $10.4000 /$ corela. 6112

ISSN : 1638-573X

Éditeur

Cercle linguistique du Centre et de I'Ouest - CerLICO

Référence électronique

Edoardo Lombardi Vallauri, «L'implicite comme moyen de persuasion : une approche quantitative », Corela [En ligne], HS-25 | 2018, mis en ligne le 09 juillet 2018, consulté le 21 décembre 2020. URL : http://journals.openedition.org/corela/6112 ; DOI : https://doi.org/10.4000/corela.6112

Ce document a été généré automatiquement le 21 décembre 2020.

\section{(c) (i) (2)}

Corela - cognition, représentation, langage est mis à disposition selon les termes de la licence Creative Commons Attribution - Pas d'Utilisation Commerciale - Partage dans les Mêmes Conditions 4.0 International. 


\title{
L'implicite comme moyen de persuasion : une approche quantitative
}

\author{
Edoardo Lombardi Vallauri
}

\section{Introduction : Implicites non linguistiques, persuasion linguistique et quantification des implicites dans les textes}

1 Transmettre un contenu de façon implicite est un procédé qui permet de réduire la possibilité pour le destinataire de remettre en question sa véracité. Cela a été décrit en détails par la linguistique récente (Givón 1982, Kerbrat-Orecchioni 1986, Rigotti 1988, Lombardi Vallauri 1993, 1995, Sbisà 2007). Cette caractéristique rend particulièrement intéressant l'emploi des implicites dans la communication à visée persuasive (Lombardi Vallauri 1995, 2009a, 2016, Sbisà 2007, De Saussure 2013, Lombardi Vallauri \& Masia 2014).

Dans les textes persuasifs, comme la publicité, l'usage de moyens visuels ou sonores non linguistiques (surtout la musique) est la règle pour transmettre des contenus implicites. Montrer un groupe d'amis riches, en bonne santé, élégants et heureux qui sauvent un cheval et puis boivent une boisson alcoolisée permet d'installer dans l'esprit du destinataire l'idée qu'il y a un lien entre boire cet alcool et être riches, élégants et heureux, sans l'affirmer. L'assertion du même contenu serait plus ridicule (ou insultante) que convaincante, tandis que dans la version implicite le message est efficace. Une musique solennelle et agréable peut être plus convaincante qu'un énoncé assertif qui dise : "cette voiture est prestigieuse et confortable". Les assertions explicites révèlent l'intention de la source de convaincre le destinataire, ce qui provoque notre volonté d'y opposer un jugement critique, en rejetant tout message qui ne soit pas complètement véridique. Au contraire, il y a beaucoup moins de réactions critiques si nous n'avons pas conscience que l'on essaie de modifier nos croyances. C'est une des raisons pour 
lesquelles image et musique supplantent les messages verbaux dans la communication publicitaire.

3 Toutefois, quand un texte persuasif doit transmettre des contenus très spécifiques, une formulation linguistique peut devenir nécessaire. Alors, pour persuader linguistiquement d'un contenu discutable, la meilleure solution est de l'encoder de façon linguistiquement implicite, et ainsi de ne pas trop s'éloigner de l'implicitation presque totale des moyens non linguistiques. Dès lors, on aimerait pouvoir quantifier l'incidence des procédés d'implicitation, particulièrement dans les textes dont les finalités persuasives ne sont pas entièrement innocentes, notamment dans la publicité et la propagande politique. Dans le présent article, nous proposerons qu'une telle quantification est possible, et nous esquisserons une possible méthode qui tienne compte soit de l'extension des implicites dans un texte, soit de leur intensité, c'est-à-dire des différents degrés de dissimulation du contenu transmis de la part de la source. Il s'agit d'une méthode sans doute encore imparfaite, mais qui a l'avantage de décomposer le processus d'évaluation en une série de phases bien définies. Un essai dans cette direction nous paraît justifié car, à l'avenir, l'application systématique d'une telle méthode à la communication publique pourrait contribuer à améliorer les conditions de démocratie réelle, dans une civilisation où les stratégies des mass media pèsent lourdement sur la formation du consensus des citoyens.

4 L'article est ainsi organize : les sections 1 et 2 distinguent entre ce que nous proposons d'appeler les implicites du contenu et les implicites de la responsabilité, à savoir entre des phénomènes comme les implicatures et le vague d'une part, et les présuppositions et les topics de l'autre. La section 3 présente la méthode proposée pour mesurer l'impact des implicites persuasifs dans les textes, et montre quelques exemples de son application à des discours politiques, qui sont comparés à des textes dont la finalité est plus informative que persuasive, et qui, comme on peut s'y attendre, obtiennent des coefficients d'implicitation beaucoup plus bas.

\section{Implicitation du contenu : les implicatures et le vague}

5 Autant avec les implicatures qu'avec le vague, une partie du contenu du message reste implicite. Les implicatures permettent au destinataire de l'inférer. Le slogan électoral utilisé par Forza Italia en 2006 montré ci-dessous n'affirmait pas que les adversaires auraient réintroduit la taxe de succession; mais les destinataires pouvaient l'inférer à cause de la maxime de relation (Grice 1975), puisqu'on était en campagne électorale, les adversaires étaient la gauche et donc en principe hostiles aux gros patrimoines :

(1) Encore une fois la taxe de succession ? Non, merci 


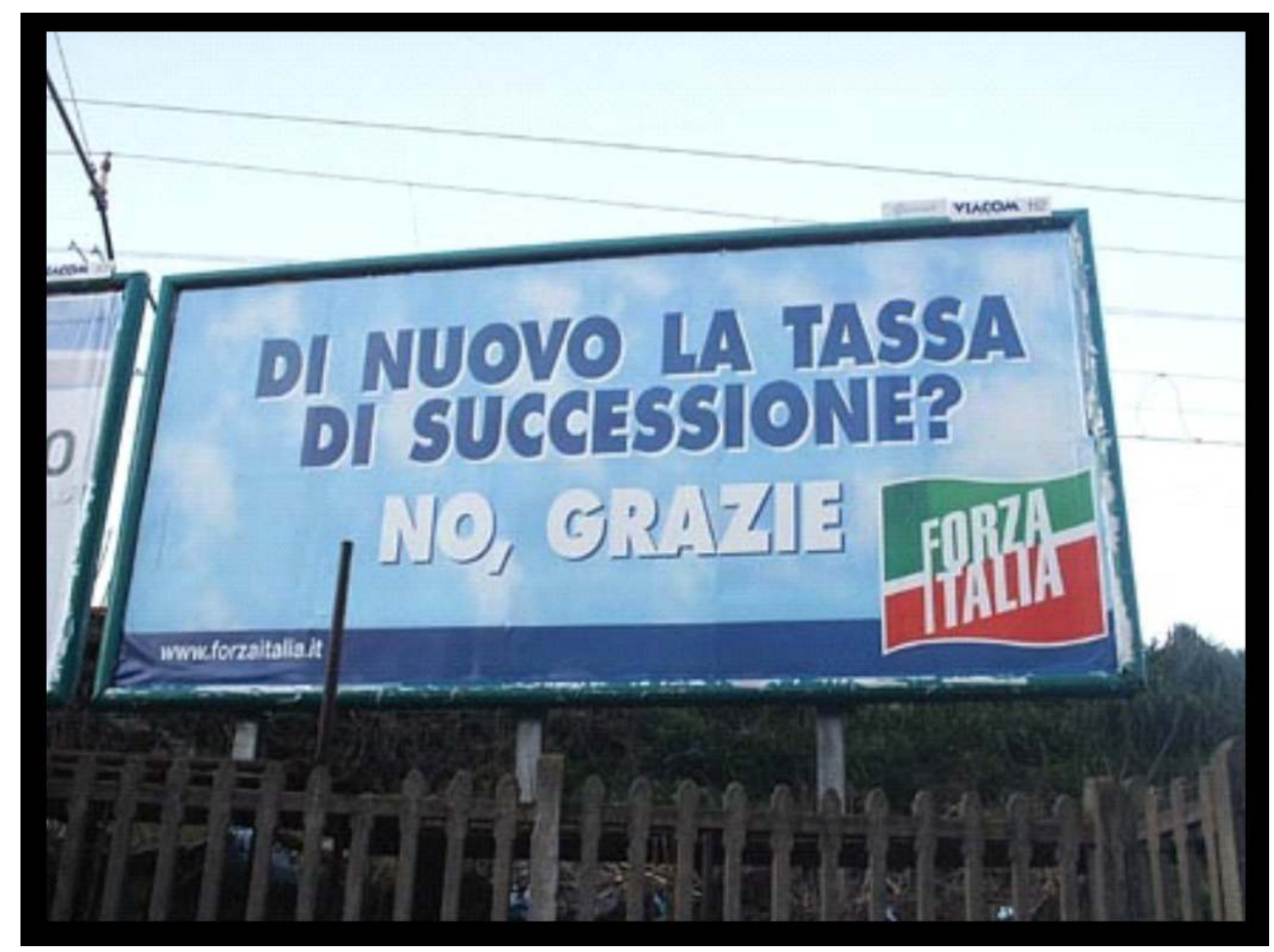

Comme c'était l'électeur lui-même qui, à l'aide d'informations partagées, construisait ce contenu, il y percevait évidemment moins une opinion exprimée par la source. La force politique qui diffusait ce message "ne l'avait pas dit", et les électeurs n'étaient donc pas amenés à le mettre en discussion. Ce contenu, à vrai dire très discutable, présenté de cette manière implicite pouvait convaincre le destinataire beaucoup mieux que si l'on avait utilisé une assertion : "La gauche va réintroduire la taxe de succession". Comme on l'a montré ailleurs, la propagande politique et la publicité commerciale font un usage systématique de cette propriété des implicatures (Lombardi Vallauri 2009a).

Le vague d'une expression est sa possibilité de se référer à plusieurs contenus (cf. Machetti 2006, Brunner 2011, Voghera 2012, pour plus de détails). Le slogan en (2) ne permettait pas de déterminer de quelle maison il s'agissait: s'agissait-il de la première, celle que l'on habite et que les politiques fiscales de gauche tendente à protéger, ou des villas de luxe qui investissent le littoral touristique? Ou bien encore des immeubles à partir desquels on tire des revenus parce qu'on les donne en location? 
(2) Plus de taxes sur ta maison ? Non, merci

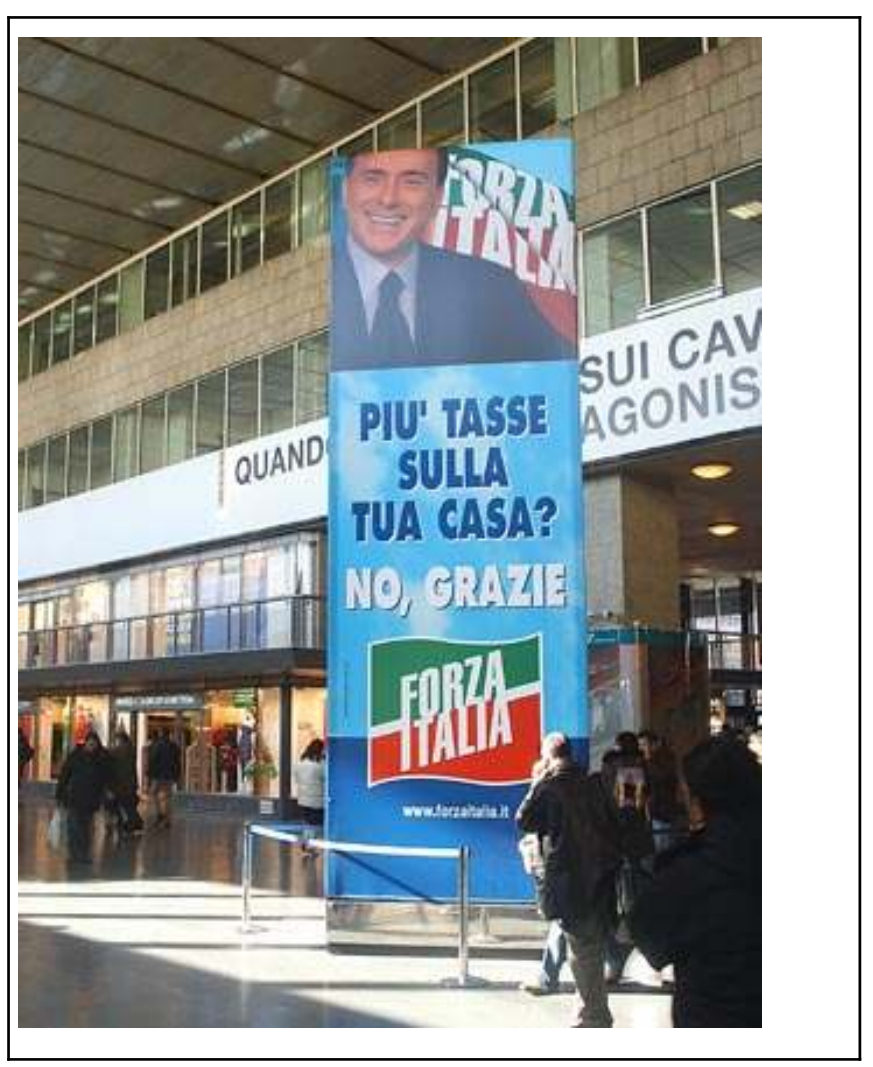

(3) La fraîcheur de Jocca a seulement 7 \% de gras

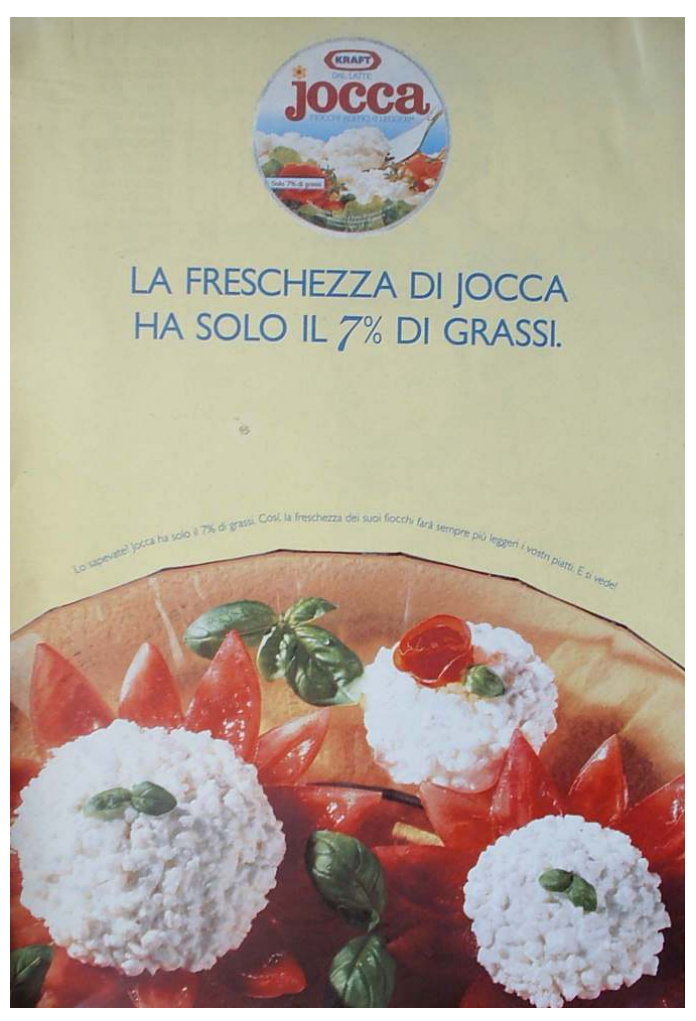


Ce contenu restait implicite. Mais chaque électeur pouvait choisir le sens qui lui convenait le mieux et qu'il trouvait moins discutable. L'idée, assez vague, que la gauche allait taxer "ta maison" s'installait plus facilement dans la vision du monde des destinataires. On peut observer le même processus interprétatif pour la "fraîcheur de Jocca", contenu transmis par la publicité en (3): le concept est tellement vague que les destinataires n'en appréhendent que la connotation positive, sans même le remettre en cause.

\section{Implicitation de la responsabilité : présuppositions et topic}

9 Un message linguistique peut encoder explicitement son contenu, tout en laissant implicite un acte de prise en charge de la part du locuteur (Lombardi Vallauri \& Masia 2014, Lombardi Vallauri 2016). On va expliquer comment cela arrive quand l'information est présentée comme présupposition ou comme topic.

\subsection{Les présuppositions}

10 La présupposition consiste à présenter un contenu comme déjà partagé par le destinataire. Dans (4), l'idée que Charles a commis un crime est encodée par une présupposition d'existence établie par l'adjectif possessif son, laquelle entretient une relation anaphorique avec un référent antérieur, en faisant allusion à sa préexistence dans le savoir partagé. ${ }^{1}$

(4) Charles est rentré à Limoges. Son crime sera puni.

11 La source présente l'idée du crime de Charles comme s'il n'était pas nécessaire de l'asserter, mais seulement de le rappeler comme présupposé, puisqu'il s'agit d'une connaissance partagée.

12 Tandis que les implicatures cachent dans l'implicite une partie du contenu, la présupposition cache le fait que celui qui produit le message est aussi la source de ce contenu, c'est-à-dire qu'elle consiste à omettre l'acte de proposer ce contenu comme vrai de la part de la source. L'énoncé (4) n'affirme pas “Charles a commis un crime”, le locuteur se présente seulement comme quelqu'un qui reprend une opinion appartenant déjà au destinataire et à d'autres gens, et ce uniquement pour donner un appui contextuel à l'assertion d'un contenu ultérieur (que Charles sera puni). Ce procédé peut distraire le destinataire du fait qu'on est en train de lui transmettre ce contenu, et par conséquent de modifier ses croyances, en attribuant à Charles un crime qui est pourtant encore à démontrer. Si le même contenu était affirmé, l'intention de transmettre ce contenu au destinataire serait beaucoup plus évidente. C'est là, à notre avis, la raison principale de ce que Givón (1982) a appelé la "unchallengeability" associée à la présupposition, dont nous allons montrer par la suite quelques conséquences.

\subsection{1. "Exaptation" des présuppositions}

13 En principe, on pourrait parler comme cela :

(5) Il existe des mois. L'un d'entre eux s'appelle février. Il existe un endroit appelé Val d'Isère. Pendant le mois que j'ai mentionné, j'irai là-bas faire du ski. J'ai une copine, et elle a des amis. C'est avec eux que j'y irai. 
Mais il est sans doute plus naturel de parler comme ceci :

(5a) En février j'irai faire du ski à Val d'Isère avec les amis de ma copine.

La préférence pour (5a) par rapport à (5) dépend du fait que son traitement exige moins d'effort cognitif. Avec toutes ses assertions, (5) pousse le destinataire à focaliser son attention sur chacun des référents qui sont introduits (les mois, février, la Val d'Isère, la copine du locuteur, ses amis), et à les traiter comme des entités qu'il ne connaît pas, en bâtissant pour chacun une nouvelle "entrée" dans sa mémoire. Sauf qu'il va tout de suite s'apercevoir qu'il connaît déjà ces référents, et qu'ils ont déjà leur place dans sa mémoire. Cet effet de garden path (Osterhout et al. 1994) est évidemment un gaspillage sur le plan cognitif. Il vaut donc mieux produire $(5 \mathrm{a})$, c'est-à-dire présenter comme déjà connus les référents que le destinataire connaît déjà (février, la Val d'Isère et le reste), et par ce moyen l'“autoriser" à ne pas les traiter de façon coûteuse, en les rappelant plutôt à l'esprit seulement comme arrière-plan dans lequel insérer le contenu affirmé (que la source ira y faire du ski).

Quand l'information est déjà connue par le destinataire, toute source communicative se doit de procéder par économie en faisant appel aux présupposés. Mais ce principe est valable aussi quand il s'agit d'information non connue par le destinataire, pourvu qu'il ne soit pas nécessaire de lui dévouer toute son attention pour comprendre le message. Par exemple, l'énoncé (6) dans lequel un verbe de changement présuppose que la porte de la cuisine est fermée, est acceptable :

(6) S'il te plaît, descends et ouvre la porte de la cuisine: je veux entendre le téléphone.

Au contraire, (6a) nous donne l'impression d'être redondant :

(6a) À présent la porte de la cuisine est fermée. Quand elle est fermée je n'entends

pas le téléphone. S'il te plait, descends et ouvre-la, je voudrais entendre s'il sonne.

L'énoncé (6), parce qu'il est plus économique (cf. Lombardi Vallauri \& Masia 2014 pour plus de détails), est préférable, bien qu'il exige une accomodation (cf. Lewis 1979), consistant à intégrer l'idée, peut-être ignorée par le destinataire, que la porte de la cuisine se trouve à présent fermée. Mais mieux vaut faire un effort d'accomodation que de focaliser toute son attention sur une information, qui est marginale. Il suffit de l'apprendre par présupposition.

9 Donc, l'essentiel "physiologique" de la présupposition est: conduire le destinataire à consacrer moins d'attention à un certain contenu, parce que la compréhension du message ne nécessite pas d'y porter plus d'attention.

Il est possible (cf. Lombardi Vallauri 2014) que la différence linguistique et discursive entre présupposition et assertion (et aussi celle entre topic et focus) s'appuie au niveau cérébral sur la différence entre automatic processing, partiellement conscient et requérant moins d'effort, et controlled processing, conscient et requérant plus d'effort. ${ }^{2}$ Les études plus récentes sur les correspondances entre activité cérébrale et structure informationnelle de l'énoncé (cf. par ex. Burkhardt 2008, Tiemann et al. 2011, Schwarz 2015, Schwarz \& Tiemann 2014), soit conduites avec les méthodes classiques de la psycholinguistique, soit avec les techniques de neuroimagerie (surtout la mesure des Potentiels Evoqués : ERP), paraissent confirmer que la même information est traitée avec moins d'effort quand elle est présupposée, plutôt que quand elle est affirmée. En perspective évolutive, on peut imaginer que la présupposition s'est développée, dans des phases anciennes de la communication linguistique, comme stratégie d'encodage 
permettant une économie d'effort cognitif lors du traitement d'une information déjà connue, et que cette possibilité a été étendue aux contenus qui ne sont pas connus mais dont le traitement en pleine attention n'est pas nécessaire à la compréhension du message (comme en (6)). Un procédé, au départ né pour une fonction différente ou moins étendue, se serait adapté à d'autres fonctions selon un processus structurellement identique à celui que, dans l'évolution du vivant, Gould \& Vrba (1982) ont appelé "exaptation".

Mais on peut concevoir un troisième stade d'exaptation des présuppositions, tiré du précédent. Présupposer un contenu inconnu du destinataire peut aussi bien servir à éviter que celui-ci devienne complètement conscient du processus de transmission. Les contenus discutables tombent sous le jugement critique du destinataire s'ils sont traités avec attention, mais ils sont au contraire acceptés plus facilement si le destinataire ne prend pas conscience de tous les détails. La même idée risque d'être rejetée quand elle est affirmée et donc soumise à la pleine attention du destinataire, beaucoup plus que quand elle est présupposée. La publicité de Philips en (7) exploite le verbe "ouvrir" pour présupposer l'idée que les destinataires vivent "les yeux fermés" :

(7) Laisse Philips t'ouvrir les yeux

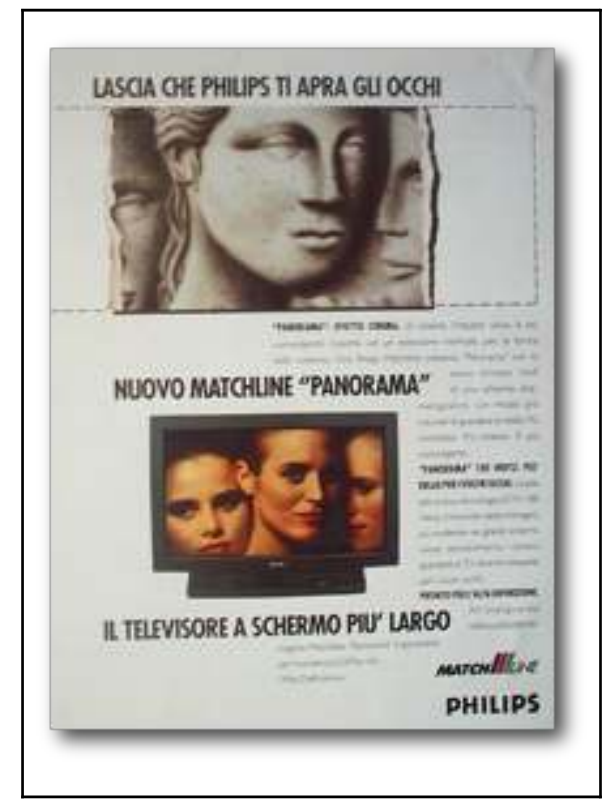

Si le même contenu était asserté, il serait peu crédible et en tout cas peu flatteur, et donc contraire aux objectifs d'un slogan publicitaire. Mais puisqu'il est présupposé, les destinataires ne concentrent leur attention que sur la suggestion (positive) de s'ouvrir à des visions agréables. Toutefois, l'idée qu'ils se trouvent dans une condition de laquelle il vaut mieux sortir va s'installer dans leur conscience, sans être soumise à un examen critique. Les présuppositions conduisent le destinataire à reconstruire paresseusement comme déjà connues et donc vraies des portions de "réalité" qui au contraire ne faisaient pas partie de ses connaissances ${ }^{3}$.

Cette stratégie caractérise amplement la publicité. Dans (8) l'assertion du père contient une présupposition qui est le contenu le plus important du message ${ }^{4}$ : 


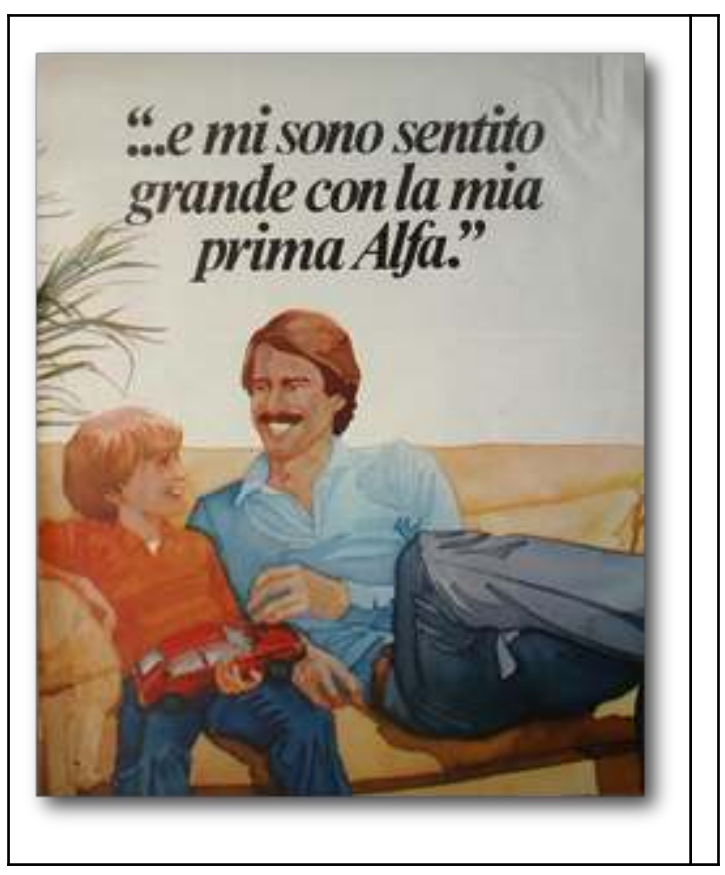

24 L'adjectif primo 'premier' présuppose que le père a continué à acheter des Alfa Romeo, d'où on peut impliquer que les Alfas sont très satisfaisantes. Si le lecteur n'est pas extrêmement méfiant, cette impression va s'installer dans son esprit, sans passer par un moment d'examen critique. L'assertion correspondante: "qui achète une Alfa en achète d'autres par la suite" serait plus difficilement acceptable.

\subsubsection{Les trois fonctions des présuppositions}

Les présuppositions ont donc trois fonctions, lesquelles découlent de la fonction générale de conduire le destinataire à moins se focaliser sur une partie de l'énoncé, et qui se distinguent selon le statut informatif de leur contenu dans la mémoire des participants :

A. épargner au destinataire l'effort superflu de traiter tel contenu comme s'il ne le connaissait pas encore, parce que ce contenu lui est déjà connu ;

B. épargner au destinataire l'effort superflu de traiter tel contenu comme n'étant pas encore connu, parce que ce contenu, quoiqu'en effet non connu, peut être traité avec moins d'attention sans nuire à la compréhension du message ;

C. empêcher le destinataire de s'apercevoir qu'une partie du contenu du message est discutable, afin qu'il ne soit pas conduit à l'examiner de façon critique et à le rejeter.

On va maintenant voir que des conditions semblables caractérisent le traitement du Topic.

\subsection{Le Topic}

L'information en topic, tout comme celle présupposée, n'est pas incorporée dans la force illocutoire de l'énoncé (cf. Cresti 2000, Lombardi Vallauri 2009b), autrement dit, elle n'est pas assertée. Tandis que la présupposition omet l'acte d'asserter un contenu en le présentant comme déjà présent dans la mémoire à long terme du destinataire, le topic 
présente son contenu comme déjà actif dans sa mémoire à court terme (cf. Chafe 1987, 1992). Autrement dit, il fait allusion à une mention récente de ce contenu dans le discours, ce qui en rend une nouvelle assertion superflue. On le mentionne donc seulement pour placer sémantiquement l'information nouvelle encodée par le focus : ce dernier porte l'illocution de l'énoncé (cf. Cresti 1992, 2000 ; Lombardi Vallauri 2001, 2009b). Si cette mention préalable a été faite par le locuteur, ce dernier va être appréhendé comme la source du contenu; mais si cela n'a pas eu lieu, le processus interprétatif sera similaire à celui décrit pour la présupposition : l'énoncé suggère au destinataire de traiter cette information comme déjà en sa possession, bien qu'en réalité elle ne le soit pas. Cela peut faciliter l'acceptation de contenus discutables.

L'exemple (9) fait mention d'une information nouvelle pour le destinataire (et en même temps décisive pour le convaincre de la qualité du produit), mais qui est présentée en topic, comme si elle avait déjà été mentionnée dans le discours. La source fait donc semblant d'en faire mention non pas pour son intérêt informationnel, mais plutôt y étant "obligée" pour "se plier" au modèle de discours qui est actif à ce moment-là :

(9) Tu dois l'écouter pour le croire

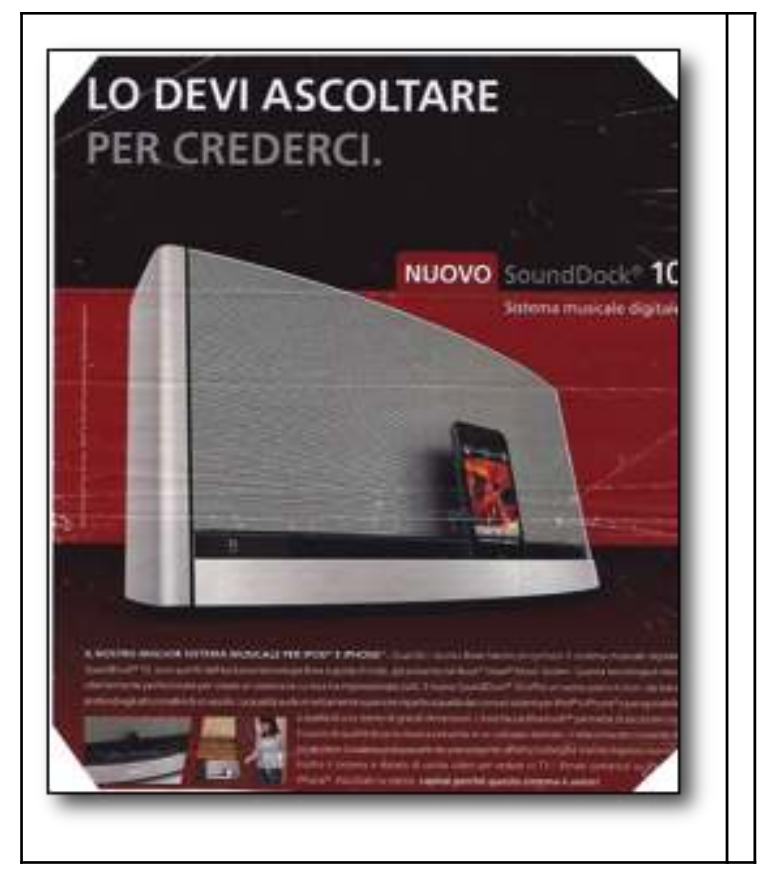

Le graphisme du titre suggère la prosodie suivante : lo devi ASCOLTARE, per crederci (tu dois 1 'ÉCOUTER, pour le croire); où la deuxième phrase est donc un topic à droite. Cela présente comme déjà active la nécessité de croire en la qualité du produit présenté ; autrement dit, on donne pour déjà partagée la croyance que la qualité du son est difficile à croire. S'il était asserté, un tel contenu paraîtrait exagéré, mais l'allusion à sa préexistence dans le contexte du discours permet de le faire accepter.

Le texte de la publicité en (10) présente comme topic de l'énoncé l'information dont on veut convaincre le destinataire (que Lindt produit un chocolat surfin), tandis que la suite rend compte d'une évidence, c'est-à-dire que cela demande un effort considérable. L'objectif est de faire accepter la première idée plus facilement, et en causant moins de réactions critiques que si elle était assertée : "nous créons un chocolat surfin". 
(10) Créer un chocolat surfin demande passion, dévouement et un grand effort...

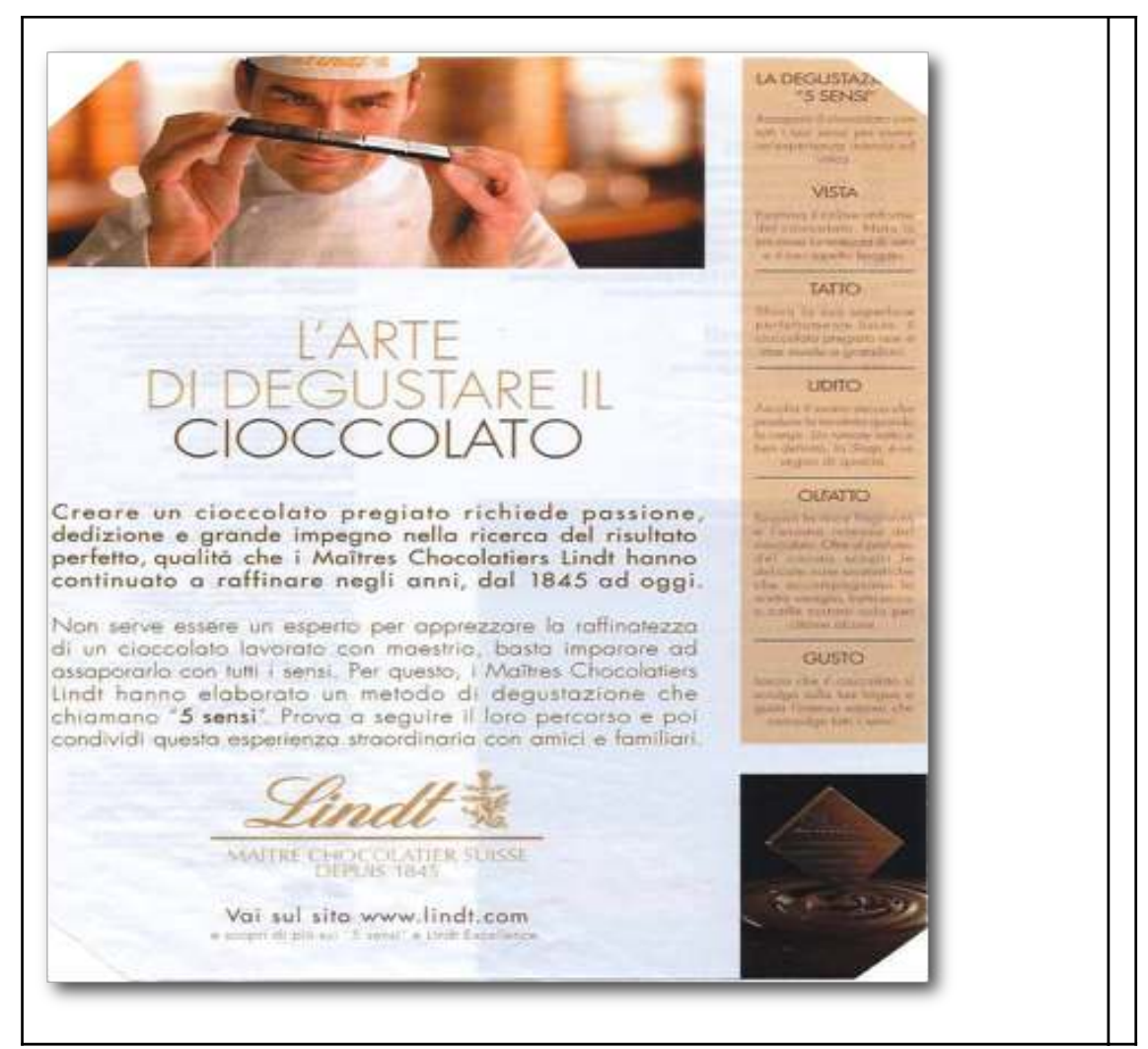

\section{Essayer de devenir quantitatifs}

On peut essayer de quantifier l'incidence des procédés d'implicitation, particulièrement dans les textes dont les finalités persuasives sont importantes, comme par exemple dans la publicité et la propagande politique.

\subsection{Ce qu'on doit compter comme "implicite sensible"}

32 Un implicite peut encoder :

i. quelque chose dont le destinataire est déjà convaincu ;

ii. quelque chose d'objectivement vrai, qu'importe si le destinataire le sait déjà ou l'ignore ;

iii. quelque chose de discutable, dont la source doit encore convaincre le destinataire.

Nous proposons d'utiliser le terme "sensible" pour caractériser les emplois du type (iii), c'est-à-dire quand la source se sert de l'implicite pour transmettre un contenu que le destinataire ne connait pas ou sur lequel il n'est pas forcément d'accord : autrement dit, quand la source évite ce que nous avons appelé la prise en charge d'un certain contenu pour soi-même, soit en le laissant impliquer par le destinataire, soit en le présentant comme déjà partagé. Il y a quelque chose d'abusif dans ce procédé, quand l'objectif est de persuader, en détournant l'attention du destinataire, d'un contenu qui ne serait pas accepté si le destinataire l'attribuait à la source et y dévouait toute l'attention nécessaire. 
Bien entendu, il est tout à fait honnête de présenter comme présupposé quelque chose de discutable, pourvu qu'on l'ait introduit auparavant de façon explicite. Notre exemple (4) ne contient plus d'implicite sensible dans la version (11) :

(11) Charles a tué mon perroquet. Maintenant il est rentré à Limoges. Son crime sera puni.

Si on voulait déterminer ce qu'on pourrait appeler "l'honnêteté comunicative"5 d'un texte, il ne serait donc pas intéressant de compter tous les implicites, mais seulement ceux du type (iii), que nous allons appeler implicites sensibles, à savoir les cas où on introduit de façon implicite un contenu qui :

- n'est pas bona fide vrai,

- n'a pas été introduit récemment dans le discours de façon assertive,

- et sur lequel les destinataires ne sont pas (tous) déjà d'accord.

Nous soutiendrons plus bas qu'une telle quantification de "l'honnêteté comunicative" d'un texte est possible, grâce à une méthode qui tienne compte soit de l'extension des implicites dans le texte, soit de leur intensité, c'est-à-dire des différents degrés de dissimulation du contenu ou de la responsabilité de la source ${ }^{6}$. Les coefficients numériques que nous allons utiliser sont arbitraires quant à leur valeur absolue, mais seule la valeur relative de chaque coefficient par rapport aux autres importe dans notre discours, car elle permettra de comparer les degrés d'exploitation des implicites dans des textes différents.

\subsection{Degrés différents d'implicite}

Parmi les implicites du contenu ${ }^{7}$, les implicatures conventionnelles représentent un degré d'implicite inférieur à celui des implicatures conversationnelles, parce que les premières ont des marqueurs lexicaux explicites :

(12) Cédric aussi aime les croissants (implicature conventionnelle: "d'autres aiment les croissants")

(13) Ce sac est beau mais économique (impl. convent.: "on s'attend que ce qui est beau soit cher")

Les implicatures conversationnelles, au contraire, ne sont pas liées spécifiquement à un élément linguistique explicite :

(14) - Jean est-il rentré de Paris?

- Je vois sa voiture devant chez lui. (impl. conversationnelle : “il est rentré”)

Les implicatures conversationnelles généralisées se trouvent en position intermédiaire, parce que les expressions linguistiques qui les activent ne le font pas directement du fait de leur sens, mais plutôt par la maxime gricéenne de quantité :

(15) Pauline a trois enfants (implicature scalaire : "elle n'en a pas plus que trois")

Pour ces raisons, nous allons assigner aux trois types d'implicatures respectivement les coefficients 1,2 et 3 (cf. Tableau 1).

Parmi les implicites de responsabilité, on peut assigner aux présuppositions dues aux descriptions définies (16), aux subordonnées adverbiales (17) et aux factifs (18) le même coefficient, s'agissant toujours du codage explicite d'un contenu, qui laisse pourtant implicite l'acte d'en assumer la responsabilité :

(16) Son yacht est le plus beau (comme tu le sais, il a un yacht)

Georges est malade (comme tu le sais, Georges existe)

Le soleil est voilé aujourd'hui (comme tu le sais, le soleil existe) 
(17) C'est ennuyeux qu'ils aient licencié Marie (comme tu le sais, ils l'ont licenciée)

Ils l'ont blâmé d'être parti (comme tu le sais, il est parti)

(18) Quand il a quitté Rome, il était chômeur (comme tu le sais, il a quitté Rome)

Il joue mieux du piano que moi (comme tu le sais, je joue du piano) même temps deux genres d'implicite. C'est le cas, par exemple, quand une présupposition véhicule une implicature, ce qui arrive en (21), contrairement à (20), où l'implicature est activée par une assertion :

(20) - Que penses-tu de la politique nationale?

- Le pays est dans une situation critique. Cela me préoccupe. (implicature par

assertion : "il faut changer de gouvernement", coefficient 3)

(21) - Que penses-tu de la politique nationale?

- La situation critique du pays me préoccupe. (implicature par présupposition: "il

faut changer de gouvernement", coefficient $3+4=7$ )

Aussi, une présupposition est plus facilement reçue quand son contenu n'est pas introduit par la source du message, et quand il est déjà connu par tout le monde : la présupposition fait alors partie du topic de l'énoncé. Déjà Bertrand Russell avait souligné la différence entre (22) et (23) :

Présupposition en topic (coefficient $4+3=7$ )

(22) The present King of France / visited the Exhibition

Présupposition en focus (coefficient 4)

(23) The Exhibition was visited by / the present King of France

Ces considérations conduisent à assigner aux genres d'implicites considérés les valeurs qu'on propose dans le Tableau $n^{\circ} 1:^{8}$

\begin{tabular}{|l|l|l|l|}
\hline Catégorie d' implicite & $\begin{array}{l}\text { Implicite de la } \\
\text { responsabilité }\end{array}$ & $\begin{array}{l}\text { Implicite } \\
\text { du contenu }\end{array}$ & $\begin{array}{l}\text { Coeff. } \\
\text { total }\end{array}$ \\
\hline (assertion explicite) & 0.0 & 0.0 & $\mathbf{0 . 0}$ \\
\hline
\end{tabular}




\begin{tabular}{|l|l|l|l|}
\hline (Focus) & 0.0 & 0.0 & $\mathbf{0 . 0}$ \\
\hline Topic & 3.0 & 0.0 & $\mathbf{3 . 0}$ \\
\hline implicature conventionnelle & 0.0 & 1.0 & $\mathbf{1 . 0}$ \\
\hline implicature généralisée & 0.0 & 2.0 & $\mathbf{2 . 0}$ \\
\hline implicature conversationnelle & 0.0 & 3.0 & $\mathbf{3 . 0}$ \\
\hline présupposition en Focus & 4.0 & 0.0 & $\mathbf{4 . 0}$ \\
\hline présupposition + Topic & $4+3=7.0$ & 0.0 & $\mathbf{7 . 0}$ \\
\hline présupposition+implicature conventionnelle en Focus & 4.0 & 1.0 & $\mathbf{5 . 0}$ \\
\hline présupposition+implicature conventionnelle+Topic & $4+3=7.0$ & 1.0 & $\mathbf{8 . 0}$ \\
\hline présupposition+implicature généralisée en Focus & 4.0 & 2.0 & $\mathbf{6 . 0}$ \\
\hline présupposition+implicature généralisée+Topic & $4+3=7.0$ & 2.0 & $\mathbf{9 . 0}$ \\
\hline présupposition+ implicature conversationnelle en Focus & 4.0 & 3.0 & $\mathbf{7 . 0}$ \\
\hline présupposition+implicature conversationnelle+Topic & $4+3=7.0$ & 3.0 & $\mathbf{1 0}$ \\
\hline condition de félicité (sans activateur explicite) en Focus & 4.0 & 3.0 & $\mathbf{7 . 0}$ \\
\hline condition de félicité (sans activateur explicite) + Topic & $4+3=7.0$ & 3.0 & $\mathbf{1 0 . 0}$ \\
\hline
\end{tabular}

Tableau $n^{\circ} 1$ : coefficients d'implicite

\subsection{Quantifier les textes}

En appliquant ces coefficients aux implicites sensibles que l'on trouve dans les textes, on obtient des valeurs différenciées selon les textes; celles-ci sont plus élevées là où les informations discutables sont présentées de façon implicite. Si un texte (fictionnaire et, en fait, impossible) était fait seulement de présuppositions de contenus sensibles, il recevrait un score total de 4 . Un texte (lui aussi impossible) fait à moitié de présuppositions et d'implicatures généralisées, pour l'autre moitié, totaliserait la moyenne entre 4 et 2 , donc 3 . Un texte composé à moitié par assertions et à moitié par présuppositions arriverait à la moyenne entre 0 et 4 , donc 2 . Un texte où les présuppositions n'occuperaient qu'un cinquième du total recevrait un score de $4 \div 5$, c'est-à-dire 0,8 .

Les scores des textes réels résultent du procédé que nous illustrons ci-dessous : dans un texte de 6600 signes, une description définie de 33 signes comme la corruption des cadres de votre parti représente $1 / 200 e ̀ m e ~ d u ~ t o t a l ~(0,005)$. Puisqu'elle présuppose son contenu, s'il s'agit d'un implicite sensible, elle reçoit un coefficient de 4 , ce qui signifie que $1 / 200 \mathrm{du}$ texte a un coefficient 4 . Si tout le texte était de la sorte, il recevrait un score de 4 ; mais 
puisque seulement une petite partie est ainsi, ce 4 pèse proportionnellement à la fraction du texte qu'il occupe : 4 x 0,005 =0,02. Cette valeur s'additionne à celle de l'implicite suivant (par exemple, 0,35 ), et ainsi de suite pour tous les implicites sensibles contenus dans le texte, jusqu'à atteindre le montant total des contributions données par tous les implicites sensibles au caractère implicite du texte.

Ce procédé, appliqué à titre d'exemple sur deux textes à visée persuasive et produits dans des circonstances similaires, a donné les résultats que nous allons présenter. Il s'agit d'un discours de Marine Le Pen (à la Baule, 26 septembre 2012) et d'un discours de Ségolène Royal (à Rennes, 4 avril 2012). Nous ne pouvons en montrer qu'un extrait pour des raisons d'espace. Les implicites sensibles sont donnés en gras :

(24) Intervention de Marine Le Pen lors de l'Université d'Eté du Front National à la Baule, 26 septembre 2012 (premiers 1867 signes) :

1. Merci à tous, chers intervenants, chers cadres et responsables du Front National,

2. militants, sympathisants, bénévoles, mes Chers Amis. Merci à tous de nous permettre

3. de clôturer aussi nombreux des universités de rentrée d'une richesse

4. exceptionnelle! Le Front National est bien la force d'avenir et d'espoir du pays, et

5. vous l'avez démontré tout au long de ce week-end!

6. Commençons mes chers amis par ces mots admirables de Michelet : «Français, de

7. toute condition, de toute classe, et de tout parti, retenez bien une chose, vous n'avez sur

8. cette terre qu'un seul ami sûr, et c'est la France! ». Or, on ne dit jamais assez, en cette

9. période où la plupart dévalorisent leur pays, en cette période où il est de bon ton de

10. se moquer de la nation, en cette période où les patriotes ont moins que d'autres la

11. possibilité de s'exprimer, on ne dit jamais assez l'amour que l'on a pour la France!

12. Vous êtes toutes et tous ici rassemblés parce que vous aimez la France.

13. Vous l'aimez d'un amour fidèle et sincère, en dépit des épreuves, en dépit des discours

14. démoralisants, en dépit de toute l'énergie que mettent beaucoup d'autres à vouloir

15. la détruire !

16. Vous êtes ici rassemblés parce que vous avez foi en un monde meilleur. Vous avez foi

17. en un monde meilleur en dépit de la désespérance dans laquelle on cherche à 18. enfermer chaque jour notre peuple. Vous avez foi en un monde meilleur parce que

19. vous savez nourrir votre espoir des mille projets que nous formons pour la France!

20. Vous êtes ici rassemblés parce que vous partagez cette passion commune qui nous

21. unit et donne un sens à notre engagement collectif, la politique !

22. Vous aimez la politique, vous aimez le débat, vous aimez le combat pour vos idéaux!

23. Parce que c'est ainsi que vous voulez changer le monde, et défendre votre pays.

24. Alors je ne m'abaisserai pas, je ne vous abaisserai pas, à vous parler de la petite 
25. politique, celle qui s'étale vulgairement tous les jours dans vos journaux, celle qui

26. au fond n'intéresse plus grand monde tant elle est méprisable.

27. Je ne vous parlerai pas de ces petites combines, de ces guéguerres, de ces spectacles

28. minables auxquels on nous donne chaque jour la peine d'assister. Les partis

29. politiques en déroute qui s'enfoncent dans la désunion et les combats de coqs, ça ne

30. m'intéresse pas!

L'impact de chaque implicite par rapport à la totalité du texte est calculé, selon les critères qu'on a expliqués, dans le tableau de calcul ci-dessous. En bas, on peut lire la valeur totale des implicites à contenu sensible dans le discours entier :

\begin{tabular}{|c|c|c|c|c|c|}
\hline A & B & C & D & E & $\mathbf{F}$ \\
\hline ligne & Texte & $\begin{array}{l}\text { genre } \\
\text { d'implicite }\end{array}$ & extension & $\begin{array}{l}\text { coefficient } \\
\text { d'intensité }\end{array}$ & $\begin{array}{l}\text { impact } \\
\text { pondéré } \\
(\mathrm{DxE})\end{array}$ \\
\hline L2-4 & $\begin{array}{l}\text { de nous permettre de clôturer aussi } \\
\text { nombreux des universités de rentrée } \\
\text { d'une richesse exceptionnelle }\end{array}$ & présupposition & 0,0175 & 4 & 0,0698 \\
\hline L4 & la force d'avenir et d'espoir du pays & présupposition & 0,0062 & 4 & 0,0246 \\
\hline L6 & ces mots admirables de Michelet & présupposition & 0,0054 & 4 & 0,0214 \\
\hline L8-11 & $\begin{array}{l}\text { cette période où la plupart } \\
\text { dévalorisent leur pays, en cette } \\
\text { période où il est de bon ton de se } \\
\text { moquer de la nation, en cette } \\
\text { période où les patriotes ont moins } \\
\text { que d'autres la possibilité de } \\
\text { s'exprimer }\end{array}$ & présupposition & 0,0329 & 4 & 0,1317 \\
\hline L11 & l'amour que l'on a pour la France & présupposition & 0,0054 & 4 & 0,0214 \\
\hline L13 & des épreuves & présupposition & 0,0022 & 4 & 0,0087 \\
\hline L13-14 & des discours démoralisants & prés+implic. & 0,0048 & $4+3$ & $\begin{array}{l}0,0190+ \\
0,0143= \\
\mathbf{0 , 0 3 3 3}\end{array}$ \\
\hline L14-15 & $\begin{array}{l}\text { toute l'énergie que mettent } \\
\text { beaucoup d'autres à vouloir la } \\
\text { détruire }\end{array}$ & présupposition & 0,0115 & 4 & 0,0460 \\
\hline
\end{tabular}




\begin{tabular}{|c|c|c|c|c|c|}
\hline L17-18 & $\begin{array}{l}\text { la désespérance dans laquelle on } \\
\text { cherche à enfermer chaque jour } \\
\text { notre peuple }\end{array}$ & prés+implic. & 0,0129 & $4+3$ & $\begin{array}{l}0,0516+ \\
0,0387= \\
\mathbf{0 , 0 9 0 3}\end{array}$ \\
\hline L19 & $\begin{array}{l}\text { des mille projets que nous formons } \\
\text { pour la France }\end{array}$ & présupposition & 0,0081 & 4 & 0,0325 \\
\hline L20-21 & $\begin{array}{l}\text { cette passion commune qui nous } \\
\text { unit et donne un sens à notre } \\
\text { engagement collectif, la politique }\end{array}$ & présupposition & 0,0159 & 4 & 0,0635 \\
\hline L23 & $\begin{array}{l}\text { que vous voulez changer le monde, } \\
\text { et défendre votre pays }\end{array}$ & Topic & 0,0093 & 3 & 0,0280 \\
\hline L24 & $\begin{array}{l}\text { Alors je ne m'abaisserai pas, je ne } \\
\text { vous abaisserai pas, à vous parler de }\end{array}$ & implicature & 0,0119 & 3 & 0,0357 \\
\hline L24-26 & $\begin{array}{l}\text { la petite politique, celle qui s'étale } \\
\text { vulgairement tous les jours dans vos } \\
\text { journaux, celle qui au fond } \\
\text { n'intéresse plus grand monde tant } \\
\text { elle est méprisable }\end{array}$ & prés+implic. & 0,0264 & $4+3$ & $\begin{array}{l}0,1056+ \\
0,0792= \\
\mathbf{0 , 1 8 4 7}\end{array}$ \\
\hline L27-28 & $\begin{array}{l}\text { ces petites combines, de ces } \\
\text { guéguerres, de ces spectacles } \\
\text { minables auxquels on nous donne } \\
\text { chaque jour la peine d'assister }\end{array}$ & présupposition & 0,0206 & 4 & 0,0825 \\
\hline L28-29 & $\begin{array}{l}\text { Les partis politiques en déroute qui } \\
\text { s'enfoncent dans la désunion et les } \\
\text { combats de coqs }\end{array}$ & prés+implic. & 0,0147 & $4+3$ & $\begin{array}{l}0,0587+ \\
0,0440= \\
\mathbf{0 , 1 0 2 8}\end{array}$ \\
\hline$\cdots$ & $\begin{array}{l}\text { (tous les implicites suivants } \\
\text { dans le texte) }\end{array}$ & $\cdots$ & $\cdots$ & $\cdots$ & $\cdots$ \\
\hline TOTAUX & & & $\underline{0,6000}$ & & $\underline{2,1758}$ \\
\hline
\end{tabular}

\section{Tableau n ${ }^{\circ}$, de calcul : discours de Marine Le Pen}

Le début du discours de Ségolène Royal est le suivant :

(25) Discours électoral de Ségolène Royal à Rennes, 4 avril 2012 (premiers 2113 signes) :

1. Chers amis, à dix-huit jours du premier tour de l'élection présidentielle, le moment est

2. venu de lancer un vibrant appel à tous ceux qui veulent s'abstenir ou qui hésitent à venir

3. voter, alors que le changement a besoin de tout le peuple de France, peuple libre,

4. peuple fier, peuple rebelle et qui mérite un nouveau destin. Oui, un nouveau destin

5. pour stopper la voracité financière sans limites qui veut imposer sa loi, pour 
6. arrêter une mauvaise gouvernance qui aggrave les injustices. Pour tout cela et pour

7. bien d'autres raisons, nous devons réussir le changement à Gauche pour tous ceux qui

8. l'attendent.

9. Et la France qui veut le changement nous appelle. Et nous lui répondons, courageux,

10. déterminés, imaginatifs, audacieux et joyeux, avec celui qui porte nos couleurs, notre

11. candidat François Hollande et dont nous devons assurer la victoire. Alors, on nous dit

12. que l'abstention deviendrait le premier parti de France, que les Français se

13. détourneraient des urnes, se sentant de moins en moins concernés. Mais vous qui êtes

14. là, si nombreux, à Rennes, venus de toute la Bretagne et d'au-delà, vous qui vous

15. mettez en mouvement, vous qui vous représentez une force citoyenne, alors, allez

16. chercher les autres, tous les autres : ceux qui se sentent écrasés, étouffés, résignés,

17. indignés, découragés, pour leur dire que si la France est en recul c'est parce que le

18. peuple a été écarté de tout. Et que si la France repart en avant, c'est parce qu'avec la

19. Gauche, le peuple sera au cœur de tout et en avant de tout ! Dites à celles et ceux qui se

30. détournent des urnes, même si leurs raisons doivent être écoutées et prises en compte,

21. de ne jamais oublier qu'à travers le monde, des hommes et des femmes risquent leur vie

22. pour pouvoir voter. Et comment ne pas, à titre d'exemple, au moment où nous sommes

23. rassemblés, comment ne pas saluer ici la victoire en Birmanie de la combattante Aung

24. San Suu Kyi? Oui, Aung San Suu Kyi, celle qui n'a jamais rien lâché face à la junte

25. militaire au pouvoir et qui en a payé le prix fort, quinze ans de réclusion et d'isolement

26. forcé. Elle nous a rappelé avant-hier que rien n'aurait été possible sans la fidélité

27. populaire. Elle a annoncé une ère nouvelle dans laquelle le rôle du peuple sera renforcé,

28. la démocratie approfondie et la pauvreté en recul. Alors nous qui avons la chance de

29. vivre en démocratie, prenons exemple sur les combats de ceux - et ils sont de plus en

30. plus nombreux à se lever à l'échelle de la planète -, prenons exemple sur les combats

31. de ceux qui veulent accéder à cette démocratie et allons voter. même procédé de calcul (que nous ne pouvons pas montrer ici en détails) donne les résultats qu'on peut lire dans les tableaux suivants, respectivement pour l'extension des implicites sensibles et pour leur impact pondéré tenant compte de leurs intensités, dans les deux discours : 


\begin{tabular}{|l|l|l|l|}
\hline & $\begin{array}{l}\text { Marine } \\
\text { Pen }\end{array}$ & $\begin{array}{l}\text { Ségolène } \\
\text { Royal }\end{array}$ & $\begin{array}{l}\text { Différences entre MLP et } \\
\text { SR }\end{array}$ \\
\hline $\begin{array}{l}\text { Extension } \\
\text { responsabilité }\end{array}$ implicitation de & $0,3851 \approx 39 \%$ & $0,0980 \approx 10 \%$ & $\mathbf{0 , 2 8 7 1} \approx \mathbf{2 9} \%$ \\
\hline$\underline{\text { Extension : implicitation du contenu }}$ & $0,2149 \approx 21 \%$ & $0,0748 \approx 7 \%$ & $\mathbf{0 , 1 4 0 1} \approx \mathbf{1 4} \%$ \\
\hline $\begin{array}{l}\text { Extension totale de l'implicite dans le } \\
\text { texte }\end{array}$ & $0,6000 \approx 60 \%$ & $0,1728 \approx 17 \%$ & $\mathbf{0 , 4 2 7 2} \approx \mathbf{4 3} \%$ \\
\hline
\end{tabular}

Tableau $n^{\circ}$ 3. Extension des implicites sensibles (en $\%$ sur le texte entier)

\begin{tabular}{|l|l|l|l|}
\hline & $\begin{array}{l}\text { Marine Le } \\
\text { Pen }\end{array}$ & $\begin{array}{l}\text { Ségolène } \\
\text { Royal }\end{array}$ & $\begin{array}{l}\text { Différences entre MLP et } \\
\text { SR }\end{array}$ \\
\hline $\begin{array}{l}\text { Impact: } \\
\text { responsabilité }\end{array}$ implicitation & $1,5312 \approx 1,5$ & $0,3836 \approx 0,4$ & $\mathbf{1 , 1 4 7 6} \approx \mathbf{1 , 1 5}$ \\
\hline$\underline{\text { Impact: } \text { implicitation du contenu }}$ & $0,6446 \approx 0,6$ & $0,2243 \approx 0,2$ & $\mathbf{0 , 4 2 0 3} \approx \mathbf{0 , 4}$ \\
\hline $\begin{array}{l}\text { Impact total de la communication } \\
\text { implicite }\end{array}$ & $2,1758 \approx 2,2$ & $0,6079 \approx 0,6$ & $\mathbf{1 , 5 6 7 9} \approx \mathbf{1 , 6}$ \\
\hline
\end{tabular}

Tableau $n^{\circ}$ 4. Impact pondéré (extension par intensité) des implicites sensibles

Pour exprimer des contenus sensibles, Le Pen a recours $43 \%$ plus souvent ( $60 \%$ à $17 \%$ ) que Royal aux implicites, soit de la responsabilité ( $29 \%$ de plus) soit du contenu ( $14 \%$ de plus). Quant à l'impact pondéré, qui s'obtient en multipliant l'extension des implicites par leurs coefficients, au total Le Pen (avec 2,2) dépasse Royal $(0,6)$ d'environ quatre fois.

De telles valeurs prennent sens aussi par la comparaison avec d'autres textes, soit du même genre que d'autres. Le 2,2 de Le Pen montre que son discours est beaucoup plus tendancieux (du point de vue de l'usage des implicites) que le texte de promotion touristique sur Internet des châteaux écossais dont nous montrons le début ci-dessous, et dont le score de 0,48 le place au contraire très peu en-dessous de Ségolène Royal :

\section{(26) Scottish Castles}

From magnificent city landmarks to mysterious ruins, Scotland is renowned for its iconic castles. Whether you are looking to explore the largest in the country, follow a regional trail or seek out those hidden gems, there is plenty of history to uncover as each castle has a fascinating tale to tell. Show less Head to Aberdeen City and Shire where you will find Scotland's only designated Castle Trail boasting 17 highlights to visit. Dunnottar Castle is perched on a cliff-top, a beautiful fortress, while Craigievar is a fine example of Scottish baronial architecture and the striking curtain wall at Balvenie will leave you awe-inspired. Scotland's capital is home to Edinburgh Castle, which overlooks the city from an extinct volcano. See the nation's crown jewels before admiring some of the oldest Renaissance decorations in Britain in The Great Hall. Step back to a time of James V at Stirling Castle's recently refurbished Royal Palace and experience a world of lavish colour 
with rich and elaborate decor. The castle also houses some beautiful tapestries and you can experience what was once a bustling kitchen as well as a Regimental Museum. Eilean Donan Castle, by the picturesque village of Dornie on the main route to Skye, is one of the most admired castles in Scotland. Ruined in a Jacobite rising, it has now been restored and is the base of Clan McRae.

L'introduction des Cambridge Examination Papers est pourtant moins tendancieuse, en ne totalisant qu'un impact de 0,06 :

(27) Introduction to Cambridge Examination Papers (2005 : v-vi) ${ }^{9}$

Paper 3. Use of English. This paper consists of five parts and tests control of English grammar, vocabulary and spelling. There are 65 questions in total. The tasks include gap-filling exercises, sentence transformation, word formation and error correction.

Paper 4. Listening. This paper contains four parts. Each part contains a recorded text or texts and some questions including multiple choice, sentence completion, true/false and matching. Each text is heard twice. There is a total of 30 questions.

Paper 5. Speaking. This paper consists of four parts. The standard test format is two candidates and two examiners. One examiner takes part in the conversation, the other examiner listens and gives marks. Candidates will be given photographs and other visual material to look at and talk about. Sometimes candidates will talk with the other candidates, sometimes with the examiner and sometimes with both.

Ailleurs (Lombardi Vallauri \& Masia 2014, 2016), nous avons mesuré les discours électoraux d'autres politiciens, américains et italiens, avec les résultats que l'on peut lire ci-dessous, comparés à ceux que nous venons d'analyser dans une sorte de classement, bien entendu tout à fait provisoire :

- Marine Le Pen (2012) = 2,1758 $\approx 2,2$

- Mitt Romney $(2012)=1,6247 \approx 1,6$

- Silvio Berlusconi (2014) = 1,1695 $\approx \mathbf{1 , 1 7}$

- Matteo Renzi (2013) = 0,9642 $\approx 1$

- Rick Santorum (2012) $=1,0500 \approx 1$

- Ségolène Royal $(2012)=0,6079 \approx 0,6$

- Enrico Letta $(2013)=0,5860 \approx \mathbf{0 , 6}$

- Dépliant promotionnel Erbolario (italien, 3.452 car.) : 2,28

- Dépliant touristique Scottish Castles (anglais, 1.802 car.) : 0,49

- Notice d'utilisation d'un médicament (italien, 2.748 car.) : 0,17

- Introduction to Cambridge Examination Papers (anglais, 2.439 car.) : 0,06

\section{Conclusion}

61 Les implicites linguistiques sont un moyen par lequel on peut soustraire des contenus sensibles (c'est-à-dire potentiellement discutables car non forcément partagés) à l'attention et à l'examen critique des destinataires, en les amenant à modifier leurs propres croyances sans s'en apercevoir. On peut essayer de mesurer l'incidence de cette stratégie dans des textes différents, en les classant ainsi sur une échelle concernant les degrés d'implicitation de l'information sensible. Ce procédé sera valide surtout entre textes comparables en termes de conditions de production et fins pragmatiques.

62 La mise au point d'un système de mesure plus fiable que la simple intuition (quoique du linguiste expérimenté) permet de formuler des jugements comparatifs moins subjectifs sur la transparence de la communication adoptée par les orateurs politiques, les campagnes électorales et de commercialisation, etc. En décomposant le processus 
d'évaluation en une série de passages bien définis comme ceux que nous avons proposés, il devient possible de reconstruire - et donc de discuter et améliorer - la façon dont on est arrivé à associer une valeur numérique à un texte: cette valeur va exprimer l'extension et l'intensité (l'impact) des implicites du contenu et de la responsabilité véhiculant des informations sensibles, c'est-à-dire ni bona fide vraie, ni partagée par les destinataires, ni introduite par la source auparavant de façon assertive et vraiment explicite.

La méthode qu'on a proposée est bien sûr perfectible, mais elle essaie de formuler une tentative de mode opératoire. À l'avenir, l'application systématique d'une telle méthode à la communication publique pourrait contribuer à améliorer les conditions de démocratie réelle, dans une civilisation où les stratégies des mass media pèsent lourdement sur la formation du consensus des citoyens.

\section{BIBLIOGRAPHIE}

BRUNNER P. (2011), Le vague - De l'usage évaluatif d'un terme en français et en allemand à la reconstruction d'un concept, Thèse de doctorat, Université Paris 3.

BURKHARDT P. (2008), Two Types of Definites : Evidence for Presupposition Cost, in GRøNN A. (ed.), Proceedings of SUB12, Oslo, Oslo University, p. 66-80.

CHAFE W. (1987), Cognitive Constraints on Information Flow, in TOMLIN R. (ed.), Coherence and Grounding in Discourse, Amsterdam - Philadelphia, Benjamins, p. 21-51.

CHAFE W. (1992), Information Flow in Speaking and Writing, in DOWNING P., LIMA S-D. \& NOONAN M. (eds.), The Linguistics of Literacy, Amsterdam - Philadelphia, Benjamins, p. 17-29.

CRESTI E. (1992), Le unità d'informazione e la teoria degli atti linguistici, in GOBBER G. (ed.), Atti del XXIV Congresso internazionale di studi della Società di Linguistica Italiana. Linguistica e pragmatica, Roma, Bulzoni, p. 501-529.

CRESTI E. (2000), Corpus di italiano parlato, Firenze, Accademia della Crusca.

DUCROT O. (1972), Dire et ne pas dire, Paris, Hermann.

FREGE G. (1892), Über Sinn und Bedeutung, in Zeitschrift für Philosophie und philosophische Kritik, n 100, p. 25-50. Rep. in IDEM, Kleine Schriften, Hildesheim, George Olms, 1967, p. 143-166. GARNER R. (1971), Presupposition in Philosophy and Linguistics, in FILLMORE C-J. \& LANGENDOEN T-D., Studies in Linguistic Semantics, New York, Holt, Rinehart and Winston, p. 22-42.

GIVÓN T. (1982), Evidentiality and Epistemic Space, Studies in Language, nº 6, 1, p. 23-49.

GRICE H-P. (1975), Logic and Conversation, in COLE P. \& MORGAN J-L., (eds.), Syntax and Semantics, vol. 3, Speech Acts, New York, Academic Press, p. 41-58.

GOULD S-J. \& VRBA E-S. (1982), Exaptation - a missing term in the science of form, Paleobiology, $\mathrm{n}^{\circ} 8$ (1), p. 4-15.

KERBRAT-ORECCHIONI C. (1986), L'Implicite, Paris, Armand Colin. 
LEWIS D. 1979, « Scorekeeping in a language game », Journal of Philosophical Logic, $n^{\circ} 8$, p. 339-359.

LOFTUS E-F. (1975), Leading Questions and the Eyewitness Report, Cognitive Psychology, $\mathrm{n}^{\circ} 7$, p. 550-572.

LOMBARDI VALLAURI E. (1993), Clausole a contenuto presupposto e loro funzione discorsiva in italiano antico, Quaderni del dipartimento di Linguistica dell'Università di Firenze, n 4, p. 71-95. LOMBARDI VALLAURI E. (1995), Tratti linguistici della persuasione in pubblicità, Lingua Nostra, $\mathrm{n}$ - 2/3, p. 41-51.

LOMBARDI VALLAURI E. (2000), Grammatica funzionale delle avverbiali italiane, Roma, Carocci. LOMBARDI VALLAURI E. (2001), The role of discourse, syntax and the lexicon in determining focus nature and extension, Linguisticae Investigationes, $n^{\circ} 23,2$, p. 229-252.

LOMBARDI VALLAURI E. (2009a), Grice elettorale, in FATIGANTE M., MARIOTTINI L. \& SCIUBBA E. (eds.), Linguistica e Società. Studi in onore di Franca Orletti, Milano, Franco Angeli, p. 172-184. LOMBARDI VALLAURI E. (2009b), La struttura informativa. Forma e funzione negli enunciati linguistici, Roma, Carocci.

LOMBARDI VALLAURI E. (2014), From the knowledge of language to the knowledge of the brain, Italian Journal of Cognitive Sciences, $\mathrm{n}^{\circ} 1,1, \mathrm{p} .131-161$.

LOMBARDI VALLAURI E. (2016), The "exaptation" of linguistic implicit strategies, SpringerPlus, n 5 (1), p. 1-24.

LOMBARDI VALLAURI E. \& MASIA V. (2014), Implicitness Impact : Measuring texts, Journal of Pragmatics, $n^{\circ} 61$, p. $161-184$.

LOMBARDI VALLAURI E. \& MASIA V. (2015), Cognitive Constraints on the Emergence of Topic-Focus Structure in Human Communication, in CHIERA A. et GANFI V. (éds), Immagine e pensiero. Bilanci nelle scienze cognitive attuali, Roma-Messina, Corisco, p. 180-204.

LOMBARDI VALLAURI E. \& MASIA V. (2016), Specificità della lingua persuasiva : l'implicito discutibile, in RUFFINO G. \& CASTIGLIONE M. (éds.), La lingua variabile nei testi letterari, artistici e funzionali contemporanei, Firenze, Cesati, p. 637-654.

MACHETTI S. (2006), Uscire dal vago, Roma-Bari, Laterza.

MOESCHLER J. (à paraître), « Présupposition et implicature : où passe la frontière ? ", dans BIGLARI A. \& BONHOMME M. (éds.), La Présupposition entre théorisation et mise en discours, Paris, Garnier.

OSTERHOUT L. \& HOLCOMB P-J., \& SWINNEY D-A. (1994), Brain potentials elicited by garden-path sentences : evidence of the application of verb information during parsing, Journal of Experimental Psychology : Learning, Memory and Cognition, $n^{\circ} 20(4)$, p. 786-803.

RIGOTTI E. (1998), « Significato e senso », Ricerche di semantica testuale, Brescia, La Scuola, p. $71-120$.

DE SAUSSURE L. (2013), Background relevance, Journal of Pragmatics, n5 59, p. 178-189.

SBISA M. (2007), Detto non detto. Le forme della comunicazione implicita, Roma-Bari, Laterza. SCHNEIDER W. \& SHIFFRIN R-M. (1977), Controlled and automatic human information processing : I. Detection, search, and attention, Psychological Review, $n^{\circ}$ 84, p. 1-66. 
SHIFFRIN R-M. \& SCHNEIDER W. (1984), Automatic and Controlled Processing Revisited, Psychological Review, n 91, 2, p. 269-276.

SCHWARZ F. (2015), " Presuppositions vs. Asserted Content in Online Processing ", in SCHWARZ F. (ed.), Experimental Perspectives on Presuppositions, Berlin, Springer's Studies in Theoretical Psycholinguistics Series, p. 89-108.

SCHWARZ F. \& TIEMANN S. (2014), Presupposition Projection in Online Processing, http://

florianschwarz.net/wp-content/uploads/papers/PresupProjectionProcessing.pdf.

STRAWSON P-F. (1964), Identifying Reference and Truth-Values, Theoria, $n^{\circ}$ 30, 2, p. 96-118. Rep. in IDEM, Logico-Linguistic Papers, London, Methuen, 1971, p. 75-95.

TIEMANN S., SCHMID M., ROLKE B., ACKERMANN H., KNAPP J. \& BECK S. (2011), Psycholinguistic Evidence for Presuppositions : On-line vs. Off-line Data, in REICH I. HORCH E. \& PAULY D. (eds.), Proceedings of Sinn \& Bedeutung 15, Saarbrücken, Universaar - Saarland University Press, p. 581-597.

VOGHERA M. (2012), Chitarre, violini, banjo e cose del genere, in THORNTON A-M. \&VOGHERA M. (a cura di), Per Tullio De Mauro, Roma, Aracne, p. 429-460.

\section{Sites Internet}

Silvio Berlusconi : http://www.youtube.com/watch?v=sCmh3wFpXjE

Marine Le Pen : http://www.frontnational.com/videos/udt-2012-la-baule-intervention-demarine-le-pen/

Enrico Letta : http://www.youtube.com/watch?v=uuQ3QaB8rII

Matteo Renzi : http://www.youtube.com/watch?v=DAM4rYp1o6w

Mitt Romney : http://www.washingtonpost.com/blogs/post-politics/post/mitt-romneys-floridarepublican-primary-speech-full-text/2012/01/31/gIQA8tYKgQ_blog.html

Ségolène Royal : http://blogs.mediapart.fr/blog/jean-marie-padovani/040412/discours-desegolene-royal-rennes

Rick Santorum : http://www.washingtonpost.com/blogs/election-2012/post/rick-santorumsmichigan-primary-speech-full-text-and-video/2012/02/28/gIQAtFsJhR_blog.html

Erbolario : http://www.erbolario.com/filemanager/img/pdf/frutto-della-passione.pdf Scottish Castles : http://www.visitscotland.com/about/history/castles/

\section{NOTES}

1. "Shared knowledge", d'après les mots de Strawson (1964). Cf. aussi Garner (1971), Moeschler (à paraître).

2. Ces deux genres de traitement cérébral sont décrits par Shiffrin \& Schneider $(1977,1984)$.

3. Loftus (1975 : 572) suggère qu'elles servent à "introduce information without calling attention to it".

4. L'implicite associé à un adjectif de quantification comme "premier" peut aussi bien être considéré une implicature scalaire ; mais cela ne change pas la substance de notre discours.

5. En parlant d'honnêteté comunicative, nous nous référons bien entendu strictement à la nature du procédé linguistique, non pas à une honnêteté plus générale, concernant le droit qu'on a de transmettre ou promouvoir tel ou tel autre contenu, dans les diverses circonstances de la vie publique et privée. 
6. Il n'est pas possible de justifier exhaustivement les critères qu'on va proposer dans l'espace accordé à cet article. Pour une présentation plus complète, cf. Lombardi Vallauri \& Masia (2014).

7. On n'a pas ici l'espace pour traiter les questions concernant les expressions vagues (cf. Lombardi Vallauri 2016).

8. On verra tout à l'heure, dans des textes réels, des exemples concrets des catégories présentées dans le Tableau $n^{\circ} 1$.

9. Cambridge First Certificate in English 7 : student's book, Cambridge University Press, Cambridge, 2005.

\section{RÉSUMÉS}

Parmi les différents types d'implicite, les implicatures portent sur le niveau du contenu (implicitation du contenu énonciatif), les présuppositions et les topicalisations sur le niveau de la responsabilité (implicitation de la responsabilité énonciative), en provoquant ce que Givón (1982) appelle la unchallengeability d'une information, c'est-à-dire la difficulté pour le destinataire d'y appliquer un "défi" cognitif qui puisse aboutir au doute sur sa vérité. Cette fonction des implicites se trouve de façon massive dans les textes persuasifs. L'article vise à quantifier l'implicitation du contenu et de la responsabilité dans quelques textes de propagande politique et commerciale, au moyen d'un système d'indices d'implicitation assignés aux dites catégories (Lombardi Vallauri \& Masia 2014). On propose ce procédé comme un exemple du rôle que l'analyse linguistique peut jouer pour l'amélioration des conditions de transparence et de garantie dans la pratique de la communication commerciale, et dans les processus où se forme le consentement démocratique.

Among linguistic implicits, implicatures leave implicit part of the content expressed by utterances, while presuppositions and topicalizations conceal the speaker's responsibility for the introduction of that content. They both effect what Givón (1982) calls the unchallengeability of some information, i.e. its being difficult to critically evaluate and reject on the part of the addressee. This function of implicits extensively characterizes persuasive texts. The paper aims at quantifying the implicitation of contents and speaker responsibility in some political and commercial advertisements, by means of a system of indexes attributed to the mentioned categories (Lombardi Vallauri \& Masia 2014). This proceeding is proposed as an example of how linguistic analysis can contribute to the improvement of fairness in commercial advertising and in political propaganda, which is at the basis of democratic cohabitation.

\section{INDEX}

Mots-clés : implicite, présuppositions, implicatures, pubblicité, propagande

Keywords : implicits, presuppositions, implicatures, advertising, propaganda 


\section{AUTEUR}

\section{EDOARDO LOMBARDI VALLAURI}

Università Degli Studi Roma Tre 